\title{
The Neural Tourniquet
}

\author{
Jason R Fritz ${ }^{1}$ and Jared M Huston ${ }^{1,2}$ \\ ${ }^{1}$ Laboratory of Biomedical Science, The Feinstein Institute for Medical Research; and ${ }^{2}$ Division of Trauma and Acute Care Surgery, \\ Department of Surgery, North Shore-LIJ Health System, Manhasset, New York, United States of America
}

\begin{abstract}
Bioelectronic medicine aims to understand and harness the beneficial effects of the nervous system and neural circuits to diagnose, treat and prevent disease. For example, targeted electrical stimulation devices can recapitulate endogenous neural signaling and control debilitating symptoms of Parkinson's disease and epilepsy. Perhaps equally as important, electrical stimulation devices demonstrate efficacy where traditional drug therapies fail. As biomedical research continues to reveal critical neural regulatory pathways, there is tremendous potential to expand and incorporate bioelectronic medicine into current treatment paradigms. In this review, we introduce a recently characterized neural pathway, termed the inflammatory reflex, which monitors and regulates systemic inflammation via afferent and efferent vagus nerve signaling. We then describe how stimulation of the vagus nerve and activation of the inflammatory reflex rapidly and specifically curtail traumatic hemorrhage through a novel mechanism termed the neural tourniquet. Finally, we identify how bioelectronic medicine can help harness the protective effects of vagus nerve signaling and the neural tourniquet to protect patients against surgical and traumatic hemorrhage.
\end{abstract}

Online address: www.bioelecmed.org

doi: 10.15424/bioelectronmed.2014.00006

\section{SURGERY AND HEMORRHAGE}

Advances in the field of surgery have revolutionized care for millions of individuals. From the development of modern cardiac surgical techniques to the removal of abdominal malignancies and repair of orthopedic fractures, the depth and scope of modern surgical practice is immense. With the more recent advent of minimally invasive laparoscopic and endovascular procedures, surgery continues to expand therapeutic boundaries while also minimizing overall pain, disability and scarring following invasive operations.

Despite these achievements, evolution within the field is marked by periods of significant patient pain and suffering. Much of this morbidity and mortality can be attributed to one of two critical obstacles that hindered progress for thousands of years. The first was management of pain in a conscious patient undergoing surgery. To minimize pain, surgeons had to operate at such a rapid pace that interventions remained limited to basic amputations and excisions, rather than more delicate and intricate procedures. Beginning in 1846, however, this obstacle was overcome when the American dentist Dr. William Morton performed a tooth extraction under ether anesthesia, and soon after Dr. John Warren performed the first successful public surgery using general anesthesia at what is now called the Ether Dome at Massachusetts General Hospital.

The second obstacle was controlling lethal surgical site infection. The risk of

Address correspondence to Jared M Huston, Laboratory of Biomedical Science, The Feinstein Institute for Medical Research; Division of Trauma and Acute Care Surgery, Department of Surgery, North Shore-LIJ Health System, Manhasset, New York, 11030. Phone: 516233-3610; Fax: 516-233-3605; E-mail: jhuston@nshs.edu.

Submitted August 24, 2014; Accepted for publication September 9, 2014; Published Online (www.bioelecmed.org) December 2, 2014.

\section{The Feinstein Institute for Medical Research Empowering Imagination. Pioneering Discovery.}

overwhelming sepsis from operative wounds long precluded anything but the most urgent and lifesaving of interventions. Approximately two decades after the successful demonstration of general anesthesia, Sir Joseph Lister spearheaded the development of surgical antisepsis with the introduction of carbolic acid to sterilize surgical wounds, instruments and even surgeons' hands. While this breakthrough was met initially with skepticism, antiseptic practices eventually led to dramatic reductions in infection-related morbidity and mortality.

Patient outcome and quality data now suggest that surgery is as safe and effective as ever (1). Notwithstanding, we contend that a third major obstacle remains to be solved. This ubiquitous but often ignored, minimized or "expected" complication is bleeding. All surgical procedures carry at least some risk of hemorrhage, even when performed by the most experienced of surgeons. Bleeding risk can range from minor wound hematomas to potentially life-threatening exsanguination. Bleeding can be lethal even without excessive blood loss, as intracerebral hemorrhage can cause death through brain herniation. 
Currently, more than 50 million inpatient surgeries are performed annually in the United States, with millions more occurring worldwide (Centers for Disease Control and Prevention [CDC], 2010 Data). The safety and efficacy of these procedures clearly depends upon surgeons' awareness of the risks of bleeding, along with the implementation of a broad therapeutic arsenal to manage intraoperative hemorrhage. Indeed, the 1912 Nobel Prize in Physiology or Medicine was awarded to the French surgeon and biologist Alexis Carrell for his pioneering work with vascular suturing techniques. Electrosurgical devices to cut tissue while limiting blood loss were introduced by Dr. William Bovie in 1926, and can now be found in just about every modern operating theater. Most recently, topical prothrombotic sealants, glues and gauzes have been introduced to aid in surgical hemostasis $(3,4)$.

If surgeons recognize the inherent and potentially lethal risk of bleeding, then the question remains why not attempt to prevent this complication before it occurs? Curiously, there are no specific therapies administered to patients before surgery to prevent hemorrhage. This stands in stark contrast to the near-universal use of pain medications, anesthesia, antibiotics and antimicrobial agents to limit pain and prevent infection, respectively. For patients receiving systemic anticoagulants or suffering from thrombocytopathies and coagulopathies, there is the option of replacing inactivated clotting factors with exogenous blood components. The vast majority of surgical procedures, however, are performed on individuals with seemingly normal hemostatic and clotting pathways.

While the majority of surgical patients maintain effective hemostasis, many do not, and a significant portion of morbidity and mortality following surgery can be attributed directly to bleeding and/or subsequent blood transfusion practices. On average, more than $20 \%$ of patients undergoing colorectal surgery require perioperative red blood cell transfusion, whereas patients undergoing primary pancreaticoduodenectomy surgery can receive up to double that number of transfusions $(5,6)$. Patients who do receive blood products are at significantly increased risk for serious perioperative morbidity and mortality $(7,8)$. Moreover, the additional health care costs associated with hemorrhagic complications are substantial (9).

Specific surgical disciplines are associated with high risks of hemorrhage.

Trauma is the fifth leading cause of death in the United States, and the number one cause of death of people under the age of $45(10,11)$. Bleeding is the most common preventable cause of death following traumatic injury. The military also faces enormous challenges to limit bleeding. Uncontrolled hemorrhage from noncompressible internal organ injury remains the leading cause of preventable soldier death on the battlefield (12). Therapies to improve hemostasis following traumatic injuries, such as administration of recombinant Factor VIIa or the antifibrinolytic agent tranexamic acid, remain limited (13-16). As a result, surgeons still rely on tourniquets, body cavity packing and holding direct pressure to manage uncontrolled traumatic hemorrhage until definitive surgical control is obtained.

\section{NEURAL REGULATION OF HOMEOSTASIS}

The central nervous system monitors and regulates homeostatic physiologic pathways through a combination of humoral and neural mechanisms. A classic humoral pathway is the hypothalamicpituitary-adrenal axis (HPA), part of the neuroendocrine system. Composed of the hypothalamus and pituitary glands in the brain and the adrenal glands in the retroperitoneum, the HPA axis monitors levels of stress, illness and physical activity and responds by releasing a combination of regulatory and counter-regulatory hormones, including vasopressin (antidiuretic hormone, $\mathrm{ADH}$ ), adrenal corticotropin hormone, cortisol and epinephrine. The HPA axis maintains diverse bodily processes including digestion, immunity and reproduction.
In addition to primarily humoralbased mechanisms, the brain modulates peripheral organs and glands through hard-wired pathways, or nerves. Perhaps the best studied is the vagus nerve (Latin, to wander), whose derivation reflects its complicated anatomical course through the body. The vagus nerve innervates organs in the thoracic and abdominal cavities, including the lungs, heart, stomach, liver, small intestine and large intestine up to the distal transverse colon. Approximately $90 \%$ of vagal fibers are afferent and transmit sensory information from the periphery to the brain. The remaining efferent fibers carry signals from the brain to peripheral organs. For example, the vagus nerve serves as the primary efferent arm of the parasympathetic nervous system, where release of acetylcholine from vagal synapses activates muscarinic receptors to slow heart rate or aid in digestion.

\section{THE INFLAMMATORY REFLEX}

It is now known that the vagus nerve forms the backbone of another brain-toimmune pathway, called the inflammatory reflex (17-19). The inflammatory reflex allows the brain to monitor and control systemic inflammatory mediators rapidly and directly. For example, afferent vagus nerve signals inform the brain of increases in systemic proinflammatory cytokine concentrations in the peritoneal cavity, often resulting from uncontrolled bacterial infection (20). The brain can then activate the efferent vagal component of the inflammatory reflex, or cholinergic antiinflammatory pathway, to downregulate production of proinflammatory cytokines $(21,22)$. In addition, the brain can activate other nonneuronal counterregulatory responses, such as fever, to help fight off potential invasive infection (23). Experimentally, activation of the cholinergic antiinflammatory pathway via electrical or mechanical stimulation of the cervical vagus nerve, or through administration of a pharmacological cholinergic agonist, inhibits proinflammatory cytokine responses and prevents lethal tissue injury in multiple 
models of systemic inflammation, shock and sepsis (24-30).

Additional studies have elucidated key anatomical, cellular and molecular components required for the antiinflammatory effects of vagus nerve stimulation. Efferent vagus nerve signaling reaches the spleen through the splenic nerve, which then increases acetylcholine release from cholineacetyltransferase-positive $\mathrm{T}$ cells $(31,32)$. Acetylcholine can inhibit proinflammatory cytokine production by stimulating the $\alpha 7$ nicotinic acetylcholine receptor subunit $(\alpha 7 \mathrm{nAChR})$ on the surface of cytokineproducing tissue macrophages (33). Interruption or removal of any of these essential components, such as through genetic deletion of $\alpha 7 \mathrm{nAChR}$ or surgical ligation of the splenic nerve, completely abrogates the protective effects of vagus nerve stimulation (31-34).

\section{THE NEURAL TOURNIQUET}

Based on the beneficial effects of the cholinergic antiinflammatory pathway, we reasoned that this mechanism could protect against other threats following traumatic injury, such as bleeding. One can argue that acute hemorrhage poses a more immediate and life-threatening insult than bacterial invasion, which requires hours or days to cause lethal tissue damage. In contrast, uncontrolled hemorrhage following injury to the central or peripheral vasculature can prove lethal in a matter of seconds to minutes.

To first explore the role of the vagus nerve in traumatic hemorrhage, we developed a peripheral soft tissue injury and hemorrhage model in swine (35). Animals receive electrical stimulation of the cervical vagus nerve or sham stimulation before standardized partial ear laceration. Compared to sham stimulation, electrical vagus nerve stimulation significantly reduces bleeding duration and volume of blood loss by $40 \%$ and $50 \%$, respectively (35). Based on the vagal contribution to cardiac function, it seemed plausible that vagus nerve stimulation might reduce bleeding by decreasing perfusion secondary to bradycardia and/or hypotension.
Nevertheless, no differences in heart rate or blood pressure of animals receiving electrical or sham vagus nerve stimulation are observed (35). This finding is in agreement with observations in mice that cholinergic antiinflammatory signaling via the vagus nerve functions at a lower activation threshold than heart rate modulation (30).

To determine if vagus nerve stimulation limits bleeding by modulating clot formation, we measured thrombin generation at the site of injury. Compared with sham stimulation, electrical vagus nerve stimulation significantly increases local thrombin-antithrombin (TAT) complex concentrations, a marker of thrombin generation (35). These differences are evident as soon as three minutes after onset of nerve stimulation, which suggests that neural pathways can regulate ongoing hemorrhage rapidly (35). In addition, there are no measurable differences in circulating TAT concentrations in vagus nerve-stimulated animals, suggesting that clotting is accelerated specifically at the site of injury (35).

We next developed a rodent hemorrhage model involving uncontrolled internal hemorrhage secondary to penetrating liver injury (36). Cholinergic stimulation is administered via the pharmacological cholinergic agonist nicotine. We observed that systemic administration of nicotine significantly and dosedependently reduces blood loss and time to cessation of hemorrhage in this model (36). Nicotine demonstrates therapeutic efficacy by significantly reducing hemorrhage when given after the onset of bleeding as well (36). We also found that nicotine significantly increases thrombin generation at the site of liver injury by more than $50 \%$ compared with vehicle-treated controls (36). There are no differences in systemic thrombin concentrations, which again suggests that cholinergic stimulation accelerates clot formation only at the site of injury (36).

\section{BIOELECTRONIC MEDICINE}

Clinicians are thoroughly familiar with organ damage caused by overwhelming systemic inflammation, which is often more dangerous to the host than the inciting stimulus. Based on more than a decade of experimental evidence, it is clear that the inflammatory reflex is critical to maintaining immunologic homeostasis. The ability of electrical vagus nerve stimulation to activate the protective effects of this neural circuit in multiple models of systemic inflammation, shock and sepsis strongly suggests that it may prove efficacious for treating critically ill patients. As such, understanding and harnessing the inflammatory reflex for clinical use fulfill the basic tenets of bioelectronic medicine.

Neural regulation of hemorrhage is a prime example of how the nervous system can help fight one of surgery's most common and difficult to treat diseases. In fact, bleeding is a problem that has existed for far longer than modern surgical time, or even human history. The threat of lethal hemorrhage following injury has plagued animals for millions of years. From an evolutionary standpoint, host defenses would have adapted to this pervasive threat by developing rapid, specific and integrated mechanisms to prevent or limit bleeding. The central nervous system is arguably the ideal modulator for such a mechanism.

The second and more practical rationale for studying neural regulation of bleeding is that multiple platforms and techniques exist to harness the nervous system's beneficial effects. One example is deep brain stimulation, which is used to treat the debilitating neurologic symptoms of Parkinson's disease (37). Deep brain stimulation has been shown to further reduce tremors, rigidity and stiffness in patients with Parkinson's disease as compared with medical therapy alone (38). In addition to targeting the brain, electrical stimulation devices can directly activate nerves, most notably the vagus nerve. In 1997, the United States Food and Drug Administration approved the use of electrical vagus nerve stimulation via an implantable pulse generator for the treatment of medically refractory epilepsy. To date, thousands of patients 
have received this therapy, and followup studies show this treatment modality to be both safe and effective with minimal side effects $(39,40)$. More recently, this therapeutic approach was approved for use in refractory depression as well (41).

From a translational perspective, vagus nerve stimulation is a promising addition to the therapeutic clinical arsenal for treating hemorrhage. While implantation of an electrical pulse generator is advantageous to treat chronic illnesses like epilepsy and depression, it is less practical for acute surgical diseases or unpredictable traumatic injuries. To this end, activation of the vagus nerve-mediated neural tourniquet may be possible through noninvasive means. Recent epilepsy studies have shown that transcutaneous auricular vagus nerve stimulation significantly reduces seizure frequency compared with baseline values (42). This intervention is based on stimulation of the Arnold nerve, the auricular branch of the vagus nerve that provides sensory innervation to the concha in the ear. Auricular transcutaneous vagus nerve stimulation has shown efficacy for treating depression as well (43). These are not the only applications of "noninvasive" neural stimulation, as transcutaneous electrical nerve stimulation (TENS) is used for the treatment of chronic pain syndromes (44).

Based on these studies, it is possible that noninvasive vagus nerve stimulation can activate the neural tourniquet. One can envision a transcutaneous nerve stimulation device available at sites throughout hospitals, including operating rooms, emergency rooms, interventional radiology and endoscopy suites, that can deliver transient, noninvasive vagus nerve stimulation to reduce the incidence of hemorrhage. The device could be attached to a patient in the pre-op waiting area, turned on for the procedure, and then removed post-operatively. Outside of the hospital, emergency medical personnel and other first responders could apply a transcutaneous stimulation device to trauma victims suffering uncontrollable hemorrhage and begin treatment immediately in the field.
Another method for activating neural circuits is pharmacologically with drugs, which remain a mainstay of treatment for many diseases. Administration of pharmacological cholinergic agonists can recapitulate the protective effects of electrical vagus nerve stimulation during inflammation (45). Moreover, cholinergic compounds are in use clinically and have favorable safety profiles (46). Administration of drugs to activate the inflammatory reflex to control bleeding would also help overcome some of the inherent limitations of invasive electrical vagus nerve stimulation, including use in emergent or urgent situations when there is no lead time to implant a stimulation device.

\section{CONCLUSION}

Modern surgical care is capable of healing a large and diverse array of diseases. Nevertheless, opportunities remain for therapeutic innovations and improvements to maximize treatment benefits and minimize unwanted side effects. Biomedical research continues to elucidate new pathways and mechanisms that protect the body against lethal pathogens and injuries. Here we describe the inflammatory reflex, an endogenous vagus nerve pathway that is positioned to limit bleeding and infection, two of the most common and dangerous sequelae of traumatic tissue injury. Bioelectronic medicine may serve as the therapeutic link between these centuries-old complications and recently characterized protective neural circuits. Restoring normal neural signaling via targeted stimulation devices may overcome the limitations of current therapeutic interventions and finally provide tangible solutions to some of surgery's most enigmatic challenges.

\section{DISCLOSURE}

The authors declare that they have no competing interests as defined by Bioelectronic Medicine, or other interests that might be perceived to influence the results and discussion reported in this paper.

\section{REFERENCES}

1. Hall BL, et al. (2009) Does surgical quality improve in the American College of Surgeons National Surgical Quality Improvement Program: an evaluation of all participating hospitals. Ann Surg. 250:363-76.

2. Number of all-listed procedures for discharges from short-stay hospitals, by procedure category and age: United States, 2010 [Internet]. [cited 2014 Sep 23]. Available from: http://www.cdc. gov/nchs/fastats/inpatient-surgery.htm. Source given as CDC/NCHS National Hospital Discharge Survey, 2010.

3. Schexneider KI. (2004) Fibrin sealants in surgical or traumatic hemorrhage. Curr. Opin Hematol. 11:323-6.

4. Gegel BT, Austin PN, Johnson AD. (2013) An evidence-based review of the use of a combat gauze (QuikClot) for hemorrhage control. AANA J. 81:453-8.

5. Qian F, et al. (2013) Variation of blood transfusion in patients undergoing major noncardiac surgery. Ann. Surg. 257:266-78.

6. Epelboym I, et al. (2014) Limitations of ACSNSQIP in reporting complications for patients undergoing pancreatectomy: Underscoring the need for a pancreas-specific module. World $J$. Surg. 38:1461-7.

7. Bernard AC, et al. (2009) Intraoperative transfusion of $1 \mathrm{U}$ to $2 \mathrm{U}$ packed red blood cells is associated with increased 30-day mortality, surgicalsite infection, pneumonia, and sepsis in general surgery patients. J. Am. Call. Surg. 208:931-7.

8. Rohde JM, et al. (2014) Health care-associated infection after red blood cell transfusion: a systematic review and meta-analysis. JAMA. 311:1317-26.

9. Stokes ME, et al. (2011) Impact of bleeding-related complications and/or blood product transfusions on hospital costs in inpatient surgical patients. BMC Health Serv. Res. 11:135.

10. Pfeifer R, Tarkin IS, Rocos B, Pape HC. (2009) Patterns of mortality and causes of death in polytrauma patients-Has anything changed? Injury. 40:907-11.

11. Hoyert DL, Xu J. (2012) Deaths: Preliminary Data for 2011. Natl. Vital Stat. Rep. 61:1-65.

12. Eastridge BJ, et al. (2012) Death on the battlefield (2001-2011): Implications for the future of combat casualty care. J. Trauma Acute Care Surg. 73:S431-7.

13. Boffard KD, et al. (2005) Recombinant Factor VIIa as adjunctive therapy for bleeding control in severely injured trauma patients: Two parallel randomized, placebo-controlled, double-blind clinical trials. J. Trauma. 59:8-18.

14. Hauser CJ, et al. (2010) Results of the CONTROL trial: Efficacy and safety of recombinant activated Factor VII in the management of refractory traumatic hemorrhage. J. Trauma. 69:489-500.

15. Dutton RP, et al. (2011) Recombinant activated Factor VII safety in trauma patients: Results from the CONTROL trial. J. Trauma. 71:12-9.

16. Shakur H, et al. (2010) Effects of tranexamic acid on death, vascular occlusive events, and blood 
transfusion in trauma patients with significant haemorrhage (CRASH-2): a randomized, placebo-controlled trial. Lancet. 376:23-32.

17. Tracey KJ. (2002) The inflammatory reflex. $\mathrm{Na}$ ture. 420:853-9.

18. Huston JM. (2012) The vagus nerve and the inflammatory reflex: wandering on a new treatment paradigm for systemic inflammation and sepsis. Surg. Infect. 13:187-93.

19. Andersson U, Tracey KJ. (2012) Neural reflexes in inflammation and immunity. J. Exp. Med. 209:1057-68.

20. Watkins LR, et al. (1995) Blockade of interleukin1 induced hyperthermia by subdiaphragmatic vagotomy: evidence for vagal mediation of immune-brain communication. Neurosci. Lett. 183:27-31.

21. Tracey KJ. (2007) Physiology and immunology of the cholinergic anti-inflammatory pathway. J. Clin. Invest. 117:289-96.

22. Huston JM, Tracey KJ. (2011) The pulse of inflammation: heart rate variability, the cholinergic anti-inflammatory pathway and implications for therapy. J. Intern. Med. 269:45-53.

23. Hansen MK, et al. (2001) The contribution of the vagus nerve in interleukin-1 beta-induced fever is dependent on dose. Am. J. Physiol. Regul. Integr. Comp. Physiol. 280:R929-34.

24. Borovikova LV, et al. (2000) Vagus nerve stimulation attenuates the systemic inflammatory response to endotoxin. Nature. 405:458-62.

25. Bernik TR, et al. (2002) Pharmacologic stimulation of the cholinergic anti-inflammatory pathway. J. Exp. Med. 195:781-8.

26. Wang H, et al. (2004) Cholinergic agonists inhibit HMGB1 release and improve survival in experimental sepsis. Nat. Med. 10:1216-21.

27. Guarini S, et al. (2003) Efferent vagal fibre stimulation blunts nuclear factor-kappaB activation and protects against hypovolemic hemorrhagic shock. Circulation. 107:1189-94.

28. Guarini S, et al. (2004) Adrenocorticotropin reverses hemorrhagic shock in anesthetized rats through the rapid activation of a vagal anti-inflammatory pathway. Cardiovasc. Res. 63:357.

29. Mioni C, et al. (2005) Activation of an efferent cholinergic pathway produces strong protection against myocardial ischemia/reperfusion injury in rats. Crit. Care Med. 33:2621-8.

30. Huston JM, et al. (2007) Transcutaneous vagus nerve stimulation reduces serum high mobility group box 1 levels and improve survival in murine sepsis. Crit. Care Med. 35:2762-8.

31. Rosas-Ballina M, et al. (2008) Splenic nerve is required for cholinergic anti-inflammatory pathway control of TNF in endotoxemia. Proc. Natl. Acad. Sci. U. S. A. 105:11008-13.

32. Rosas-Ballina M, et al. (2011) Acetylcholine-synthesizing $\mathrm{T}$ cells relay neural signals in a vagus nerve circuit. Science. 334:98-101.

33. Wang H, et al. (2003) Nicotinic acetylcholine receptor alpha7 subunit is an essential regulator of inflammation. Nature. 421:384-8.
34. Huston JM, et al. (2006) Splenectomy inactivates the cholinergic anti-inflammatory pathway during lethal endotoxemia and polymicrobial sepsis. J. Exp. Med. 203:1637-42.

35. Czura CJ, et al. (2010) Vagus nerve stimulation regulates hemostasis in swine. Shock. 33:608-13.

36. Fritz JR, Gersch RP, Huston JM. (2012) Cholinergic stimulation prevents lethal abdominal hemorrhage [Abstract]. Shock. 37 Suppl 1:93.

37. Krack P, et al. (2003) Five-year follow-up of bilateral stimulation of the subthalamic nucleus in advanced Parkinson's disease. N. Engl. J. Med. 349:1925-34.

38. Deuschl G, et al. (2006) A randomized trial of deep-brain stimulation for Parkinson's disease. N. Eng. J. Med. 355:896-908.

39. DeGiorgio CM, et al. (2000) Prospective long-term study of vagus nerve stimulation for the treatment of refractory seizures. Epilepsia. 41:1195-200.

40. Handforth A, et al. (1998) Vagus nerve stimulation therapy for partial-onset seizures: a randomized active-control trial. Neurology. 51:48-55.

41. Aaronson ST, et al. (2013) Vagus nerve stimulation therapy randomized to different amounts of electrical charge for treatment-resistant depression: acute and chronic effects. Brain Stimul. 6:631-40.

42. He W, et al. (2013) Transcutaneous auricular vagus nerve stimulation as a complementary therapy for pediatric epilepsy: a pilot trial. Epilepsy Behav. 28:343-6.

43. Hein E, et al. (2013) Auricular transcutaneous electrical nerve stimulation in depressed patients: a randomized controlled pilot study. J. Neural Transm. 120:821-7.

44. Dailey DL, et al. (2013) Transcutaneous electrical nerve stimulation reduces pain, fatigue and hyperalgesia while restoring central inhibition in primary fibromyalgia. Pain. 154:2554-62.

45. Pavlov VA, et al. (2006) Central muscarinic cholinergic regulation of the systemic inflammatory response during endotoxemia. Proc. Natl. Acad. Sci. U. S. A. 103:5219-23.

46. Satapathy SK, et al. (2011) Galantamine alleviates inflammation and other obesity-associated complications in high-fat diet-fed mice. Mol. Med. 17:599-606.

Cite this article as: Fritz JR, Huston JM. (2014) The neural tourniquet. Bioelectron. Med. 1:25-9. 